\title{
Nerve optic segmentation in CT images using a deep learning model and a texture descriptor
}

\author{
Ramin Ranjbarzadeh $^{1}$ (D) Shadi Dorosti ${ }^{2}$. Saeid Jafarzadeh Ghoushchi ${ }^{3}$. Sadaf Safavi ${ }^{4} \cdot$ Navid Razmjooy $^{5}$. \\ Nazanin Tataei Sarshar ${ }^{6}$. Shokofeh Anari ${ }^{7}$. Malika Bendechache ${ }^{8}$
}

Received: 4 September 2021 / Accepted: 11 February 2022 / Published online: 28 February 2022

(c) The Author(s) 2022

\begin{abstract}
The increased intracranial pressure (ICP) can be described as an increase in pressure around the brain and can lead to serious health problems. The assessment of ultrasound images is commonly conducted by skilled experts which is a timeconsuming approach, but advanced computer-aided diagnosis (CAD) systems can assist the physician to decrease the time of ICP diagnosis. The accurate detection of the nerve optic regions, with drawing a precise slope line behind the eyeball and calculating the diameter of nerve optic, are the main aims of this research. First, the Fuzzy C-mean (FCM) clustering is employed for segmenting the input CT screening images into the different parts. Second, a histogram equalization approach is used for region-based image quality enhancement. Then, the Local Directional Number method (LDN) is used for representing some key information in a new image. Finally, a cascade Convolutional Neural Network (CNN) is employed for nerve optic segmentation by two distinct input images. Comprehensive experiments on the CT screening dataset [The Cancer Imaging Archive (TCIA)] consisting of 1600 images show the competitive results of inaccurate extraction of the brain features. Also, the indexes such as Dice, Specificity, and Precision for the proposed approach are reported 87.7\%, 91.3\%, and 90.1\%, respectively. The final classification results show that the proposed approach effectively and accurately detects the nerve optic and its diameter in comparison with the other methods. Therefore, this method can be used for early diagnose of ICP and preventing the occurrence of serious health problems in patients.
\end{abstract}

Keywords Convolutional neural network · Image segmentation · Deep learning $\cdot$ Image enhancement $\cdot$ Nerve optic

Ramin Ranjbarzadeh

ranjbar.ramin24@gmail.com

Shadi Dorosti

Shadi.dorosti@gmail.com

Saeid Jafarzadeh Ghoushchi

s.jafarzadeh@uut.ac.ir

Sadaf Safavi

sf.safavi@gmail.com

Navid Razmjooy

navid.razmjooy@hotmail.com

Nazanin Tataei Sarshar

ab.tataee@gmail.com

Shokofeh Anari

shokofehanarii@gmail.com

Malika Bendechache

malika.bendechache@dcu.ie

1 Department of Telecommunications Engineering, Faculty of Engineering, University of Guilan, Rasht, Iran

2 Department of Industrial Engineering, Urmia University of Technology (UUT), Urmia, Iran

3 Faculty of Industrial Engineering, Urmia University of Technology, Urmia, Iran

4 Department of Computer Engineering, Mashhad Branch, Islamic Azad University, Mashhad, Iran

5 Department of Electrical Engineering, Tafresh University, Tafresh, Iran

6 Department of Engineering, Islamic Azad University, Tehran North Branch, Tehran, Iran

7 Department of Accounting, Economic and Financial Sciences, Islamic Azad University, South Tehran Branch, Tehran, Iran

8 School of Computing, Faculty of Engineering and Computing, Dublin City University, Dublin, Ireland 


\section{Introduction}

Increased intracranial pressure (ICP) is considered a substantial neurological issue that may lead to permanent neurological sequelae by growing pressure inside the skull [1]. The main reasons for ICP occurrence include blood pooling in some parts of the brain, brain tumor, cerebrospinal fluid, aneurysm, swelling in the brain, head injury, and infections such as meningitis, stroke, hydrocephalus, high blood pressure, or encephalitis $[2,3]$. The most common symptoms of an ICP are vomiting, a headache, weakness or problems with moving or talking, blurred vision, and lack of energy or sleepiness [4].

A person with a high ICP may require immediate medical treatment, to bring down the pressure on brain tissue which helps to diminish the risk of brain damage. Therefore, a robust, accurate, and reliable non-invasive strategy for measuring elevated ICP is a core building block in this field [5-7]. To diagnose ICP, the expert may review the medical history and physical exam to test senses, balance, and mental status measures the pressure of the cerebrospinal fluid through a spinal tap, use of computed tomography (CT), magnetic resonance imaging (MRI), and series of cross-sectional X-ray to detect subtle changes in brain tissue.

One of the most reliable and cost-effective approaches to diagnose ICP is the measurement of optic nerve sheath diameter (ONSD) [8-10]. The ONSD has a significant role in the assessment of papilledema in cases of elevated intracranial pressure. The ONSD alterations in thickness with cerebrospinal fluid (CSF) pressure alterations as there is a layer of subarachnoid space among the nerve and its sheath, which inflates due to increased intracranial pressure $[11,12]$. The ONSD is commonly measured $3 \mathrm{~mm}$ from the posterior globe boundary, as this is thought to be the point of maximal pressure changes along the long axis of the optic nerve; however, the results of measuring in this location are not accepted universally. These measurements can be accomplished on ultrasound utilizing a linear array probe on a CT image or a T2-weighted sequence on an MRI image [9]. The intramodality measurement difference is modest and correlates well with variations in CSF pressure.

The assessment of ONSD manually is time-consuming and subject to human errors. Consequently, a measurement approach based on automatic machine learning techniques can eliminate some potential errors [13-15]. Labeling similar regions in an input image are considered image segmentation and is a key step in the field of medical image analysis. Image segmentation is the process of partitioning an image into multiple segments and it is a typical approach is used to locate objects and boundaries in images based on similar attributes such as orientation, dimension, and color [16]. Image segmentation is generally considered an intermediate step of some pattern-recognition applications [17, 18]. Also, the segmentation procedure is utilized either as an initial or last processing step $[19,20]$.

Based on recent studies, machine learning, deep learning, and artificial intelligence techniques have found a considerable application to improving diagnosis and decision-making in brain injury disorders like ICP [21]. In the following, some papers that applied computer-based methods for better prediction, diagnosis, and estimation of ICP are mentioned.

Ojeda et al. [22] confirmed the promising result of using the deep learning model which is developed via Aidoc (Tel Aviv, Israel) to accelerate the diagnosis of ICP in patients. Raj et al. [23] proposed an algorithm based on machine learning to predict mortality after traumatic brain injury. The logistic regression modeling is utilized for this purpose and a cross-validation method is employed to evaluate the results. However, the main limitation is that the proposed logistic regression cannot include more sophisticated features and it does not provide estimates of errors for the individual predictions. Naraei et al. [24] suggested the statistical and machine learning methods to find a correlation between ICP and routinely monitored physiological signals in Traumatic Brain Injuries (TBI) patients and the main drawback of the proposed approach is its inability to analyze principal components and define components of the features. This inability causes to estimate the diameter of nerve optic inaccurately. Moreover, their method suffers from a small rate of accuracy when deal with a sample with fuzzy borders. Chen et al. [25] presented a technique to estimate the ICP level. After feature extraction, the super vector machine is used for building a prediction model. However, due to the problem of the imbalanced datasets, the efficiency of the suggested method for the large dataset is not decisive. Moreover, their method is not able to recognize the diameter of sheath in both eyes appropriately. In Lee et al. [5] paper, deep learning techniques were used to remove signal artifacts from continuous ICP control and to determine improvements in clinical parameter prognostic capacities after artifact removal. The stacked convolutional auto-encoder (SCAE) and CNN with tenfold cross-validation tests are utilized for this purpose. Although this method is capable of extracting more unique features in comparison of hand-crafted feature extraction methods, it still needs a powerful pre-processing step to enhance the quality of image before any further process. Hu et al. [26] optimized an algorithm that can effectively find the optimal subset of metrics for better classification performance via a morphological clustering approach. Quachtran et al. [27] investigated the use of Deep Learning to model the relationship between hypertension and waveform morphology with the aim of detecting hypertension presence with high accuracy. However, the proposed method applied to a small number of data and it should be tested on a dataset with a large amount of data. 
In this paper to overcome the mentioned problems, a Convolutional Neural Network model $(\mathrm{CNN})$ is proposed for nerve optic segmentation in the accurate distance behind the eyeball. We employed two distinct images (a CT scan image and an encoded image) as the input of the model. Also, the Fuzzy C-Mean (FCM) is used for segmenting the CT image into different parts. Then, a region-based image quality enhancement strategy is employed to improve the contrast and quality of the $\mathrm{CT}$ image. Moreover, to represent key features of the input image, a texture descriptor approach, namely Local Directional Number Pattern (LDN), is utilized to increase the capability of the feature extraction procedure.

\section{Methodology}

In the proposed method, to detect the nerve optic in accurate slope and distance from the back of the eyeball, a comprehensive approach is followed. Firstly, the pre-processing method was employed on the $1600 \mathrm{CT}$ screening images obtained from a public dataset [28]. Each of the CT images is clustered through the Fuzzy C-mean (FCM) to five different parts. Next, the desired region of images in the chosen cluster is improved via a histogram equalization approach. Next, Local Directional Number (LDN) as a texture descriptor technique is employed to create a new image from the improved images in the previous steps. To obtain results with high accuracy, the two images are utilized as the input images of the proposed convolution neural network (CNN) for feature extraction and classification of nerve optic from other parts of the brain. In the following, the proposed approach is explained comprehensively.

\section{Fuzzy c-means}

Fuzzy c-means (FCM) is a strategy of dividing data or pixels into distinct parts which allow one piece of data to belong to two or more clusters. This strategy was developed by Dunn in 1973 [29] and improved by Bezdek in 1981 [30]. FCM is a variant of the soft c-means clustering technique. In nonfuzzy clustering, data are divided into distinct clusters, where each data point can only belong to exactly one cluster. In fuzzy clustering, data points can potentially belong to multiple clusters. In the FCM approach, by iteratively updating the membership level of each pixel, the cluster center of each segment is searched [31-34]. The main objective of the fuzzy c-means strategy is to diminish the following objective function:

$F=\sum_{i=1}^{n} \sum_{j=1}^{m} \gamma_{i j}\left\|P_{i}-C_{j}\right\|^{2}$,

$$
\begin{aligned}
& \gamma_{i j}=1 /\left(\sum_{k=1}^{n}\left(\frac{P_{i}-C_{i}}{P_{i}-C_{k}}\right)\right)^{t}, \\
& \sum_{k=1}^{n} \gamma_{i j}=1, \gamma_{i j} \in[0,1] .
\end{aligned}
$$

In these equations, $P$ and $C$ are the abbreviations of pixel and center, respectively. The $\gamma_{i j}$ is the membership value of the $j$ th sample in $i$ th cluster and $n$ indicates some clusters and, $m$ represents some pixels $[19,35,36]$.

\section{Histogram equalization}

Enhancing the quality of input data before processing is an important step for obtaining promising results [37]. Image enhancement aims to improve the perception or interpretability of information in an image and bring out the hidden image details to increase the image contrast with a new dynamic range. Conventional histogram equalization is one of the most popular approaches due to its effectiveness and simplicity $[38,39]$. Conventional histogram equalization adjusts the contrast of an image by modifying the intensity distribution of the histogram. This strategy usually rises the global contrast of many images, especially when the usable data of the image is represented by close contrast values [40]. Through this adjustment, the intensities can be better distributed on the histogram. This allows for regions of lower local contrast to achieve a higher contrast [41]. Histogram equalization accomplishes this by effectively spreading out the most frequent intensity values $[42,43]$. The implementation of this approach is as follows:

Step 1: Calculating the probability of $i$ th pixel-level occurrence in $\{x\}$ as a grayscale image

$p_{x}(i)=p(x=i)=n_{i} / n, 0 \leq i \leq m$,

where $n_{i}$ is the number of $i$ th gray-level occurrences, $n$ represents the total number of pixels in the image and $m$ is the image's total number of gray levels [44]. Also, $p_{x}(i)$ is the image's histogram for pixel value $i$.

Step 2: Defining the normalized accumulative distribution function corresponding to $p_{x}$

$\operatorname{cdf}_{x}(i)=\sum_{j=0}^{i} p_{x}(x=j)$.

Step 3: Produce a new image $\{y\}$, with a flat histogram

$\operatorname{cdf}_{x}(i)=i R$, 
where $R$ is a constant value. The cumulative distribution function of such an image will be linearized through the value range.

Step 4: Transforming $\operatorname{cdf}_{x}(i)$ based on the $y=T(x)$ feature of the cumulative distribution function

$y=T(r)=\operatorname{cdf}_{x}(r)$,

where $r$ is in the range $[0, m]$ and $T$ maps the levels into the range $[0,1]$.

Step 5: In order to map the values back into their original range, the following transformation needs to be applied

$y^{\prime}=y \cdot(\max \{x\}-\min \{x\})+\min \{x\}$.

However, the conventional histogram equalization has some drawbacks. This technique frequently over-enhances the image's background, resulting in level saturation effects in small but visually significant areas. Image areas with higher intensity value occurrences are often highlighted in conventional histogram equalization, and these sections of the image are frequently over-enhanced [45]. Conversely, the sections of the picture with relatively small intensity values occurrence may eliminate an equalization process. To deal with the problem mentioned above, in this paper, effective region-based histogram equalization is proposed which is applied on the brain CT images.

\section{Proposed image quality enhancement}

In the proposed approach, the quality enhancement of the images in the eyeball region for better detection of the nerve optic is the initial step of this technique. For this purpose, the input images are clustered via the FCM approach to the 5 parts. Figures 1 and 2 are two examples among 1600 CT images that demonstrate the region-based enhancement approach specifically.

In Fig. 1, ' $a$ ' is the input image and ' $b$ ' is the result of clustering in five different parts based on the FCM method. In this approach, instead of total image enhancement, the main and important region of the image is improved through the histogram equalization method. Therefore, to improve a specific region of the image, among five different clusters, the clusters are chosen for image enhancement that meets some morphological criteria including (1) As the region around the eyeballs indicated minimally the $30 \%$ of the image, the area of all objects inside the image needs to be bigger than the $30 \%$ of all pixels inside the image (Fig. 1, images 1 and 4 meet this condition), (2) As eyeballs are presented next to the center of the brain, the center of the biggest object needs to be close to the center of the image (Fig. 1, images 1, 2, and 4 meet this condition), (3) the solidity of the biggest object need to be bigger than 0.6 (Fig. 1, image 1 meets this condition). The output image in the third row (section ' $c$ ') is the contrast adjusted form of the image which is modified by the intensity distribution of the histogram.

Figure 2 is another example; the input image is clustered based on the color intensities of the five different parts. Section ' $c$ ' shows the output image with enhanced quality which is the result of employing the histogram equalization approach. Overall, the region-based approach can save time and accelerate the histogram enhancement approach. Due to the textural difference in the brain tissue between individuals, there are some differences between the order and the shape of the five obtained images using the fuzzy method in the image quality enhancement step. However, as we analyze these images based on some morphological features, this problem can be solved easily.

The optic nerve starts at the optic disk (cannot be recognized in the original image), a structure that is 0.06 inch $(1.5 \mathrm{~mm})$ in diameter and is localized at the back of the eyeball. So, before extracting features to calculate the diameter of the nerve, it is essential to improve the quality of the image, especially in these regions. In the following, Fig. 3 shows further examples of input image quality enhancement through the region-based histogram equalization. As clearly demonstrated, the nerve optics behind the eyes and regions next to them are now recognizable. So, in this step, we can work on finding the nerve optic inside the image. In the next step, a texture descriptor strategy is employed to provide a new representation of the image obtained from the previous step [46].

\section{Proposed image quality enhancement}

A texture descriptor is a feature vector containing various information about an image. In this paper, the Local Directional Number (LDN) method encodes the structure of a local neighborhood by analyzing its directional information. LDN as a descriptor creates an image from components of the same type, measuring, algebraic, geometric, statistical, differential, or spatial properties of an image [47, 48].

The LDN equation can be defined as followed:

$\operatorname{LDN}(c x, c y)=\operatorname{Eight}\left(p_{c x, c y}\right)+n_{c x, c y}$,

where $(c x, c y)$ represents the mid pixel of the neighborhood and $p_{c x, c y}$ is the maximum positive. Moreover, the $n_{c x, c y}$ is the minimum value of the negative response. Figure 4 demonstrates some improved CT images based on histogram equalization and the related images produced by the LDN. It should be mentioned that in the CT images, the head and the brain are not always located in the center. There is a possibility that the direction of the brain is inclined up, down, left, or right of the image. 


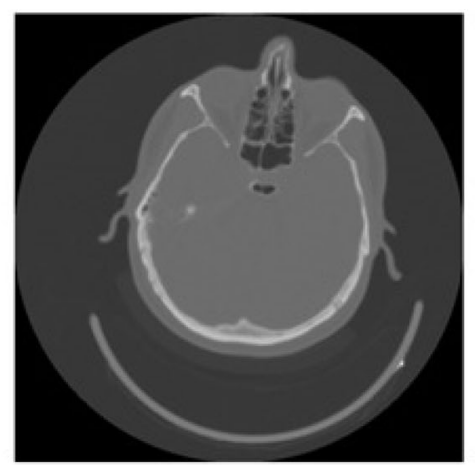

\section{b}
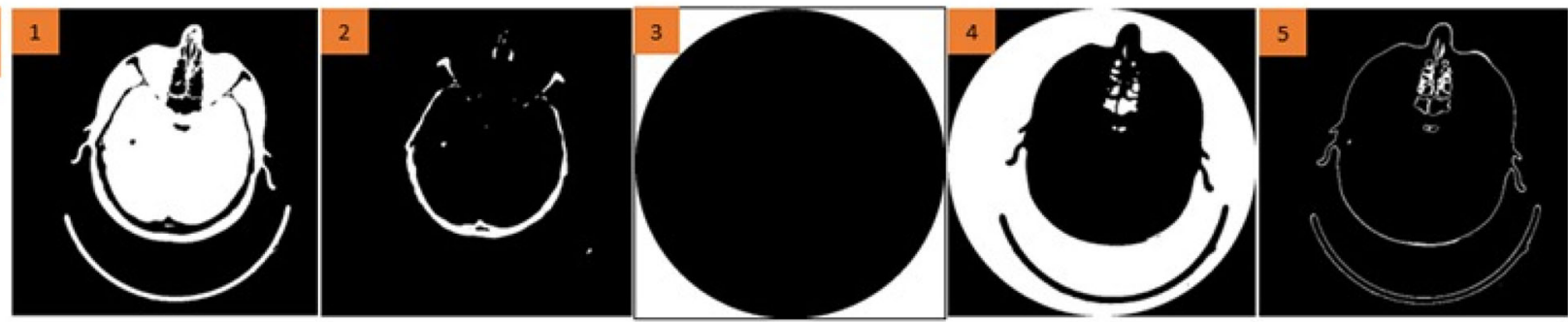

C

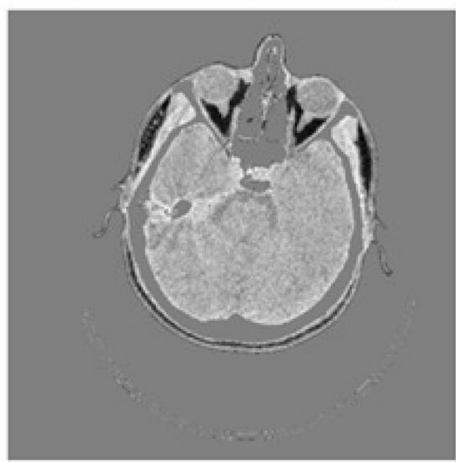

Fig. 1 An example of brain CT images. a The original image, b Five different clusters, $\mathbf{c}$ The enhanced image

\section{Proposed convolutional neural network architecture}

Convolutional neural networks (CNNs), were first introduced in the 1980s by Yann LeCun [49]. The CNNs are composed of multiple layers of artificial neurons. $\mathrm{CNN}$ is a type of deep learning structure that has a grid pattern and is employed for processing data, such as images. $\mathrm{CNN}$ is employed for adaptively and automatically learning spatial hierarchies of features via a backpropagation process using various building blocks [50-53]. This neuron-based network that has a gridlike topology, automatically extracts high-level features from raw input data whereas, their corresponding spatial information can be preserved. This structure includes some weights and biases and is applied for feature extraction, prediction, and classification. The core building block or essential part of any CNN is defined as the convolutional layer which transforms an input into a stack of feature mappings of that input. Regularly, this layer is located at the initial part of the CNN pipeline and considered as the prior layer for extracting features from an input image $[54,55]$.
CNNs can describe a mathematical construct that is typically composed of three kinds of building blocks (or layers): convolution, pooling, and fully connected layers [56]. The first two layers, convolution and pooling layers, act as feature extraction layers, whereas the third layer, a fully connected layer, maps the extracted feature matrixes into the final output, such as classification. A convolution layer is a vital part of a CNN model, which is composed of a stack of mathematical operations, such as convolution, a specialized type of linear operation [57]. The innovation of convolutional neural networks is the capacity of learning a large number of patterns in parallel automatically. This learning procedure is specified to a training dataset under the constraints of a specific predictive modeling issue, such as image segmentation, regression, or classification [58].

A convolution layer using some masks (kernels) extracts some crucial features of the texture. The kernel is a set of numbers with a smaller size than the input image. A larger kernel leads to a larger receptive field. It means it takes more 
a

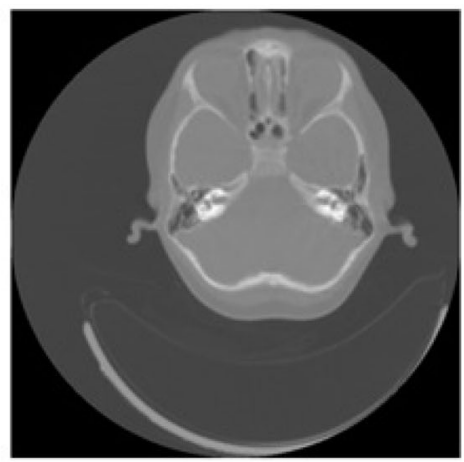

b
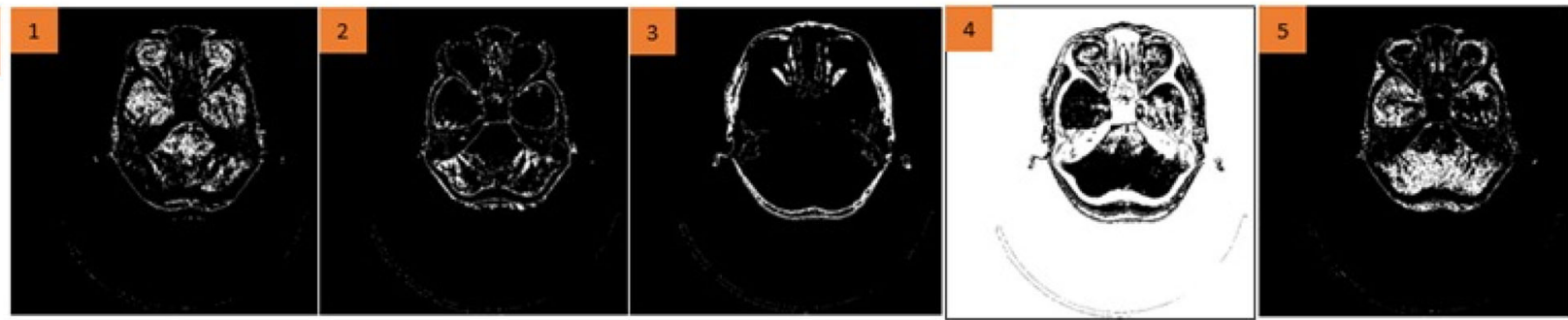

C

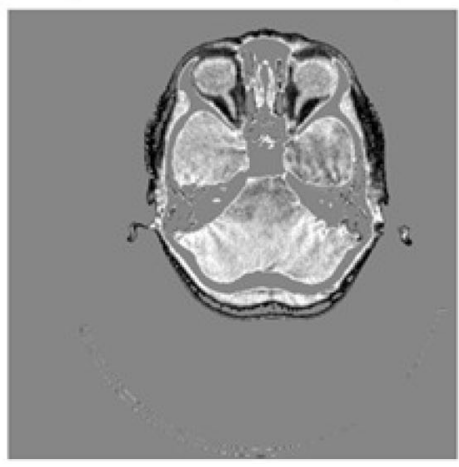

Fig. 2 An example of a brain CT image, a The original image, b Five different clusters, and $\mathbf{c}$ The enhanced image

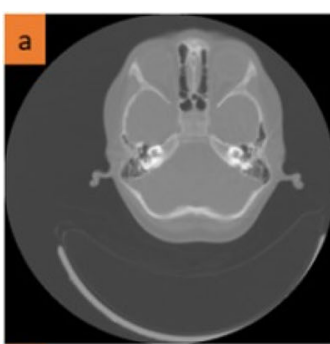

b

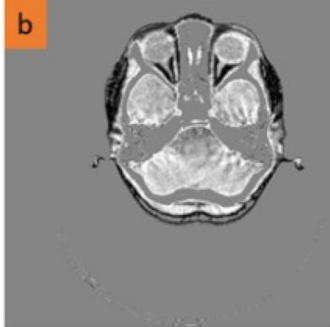

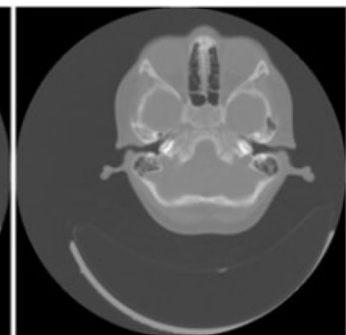

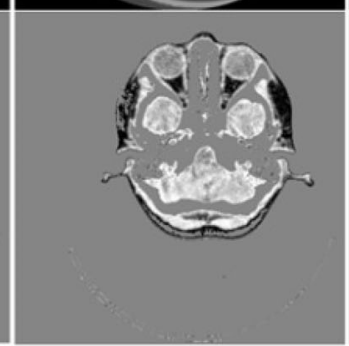

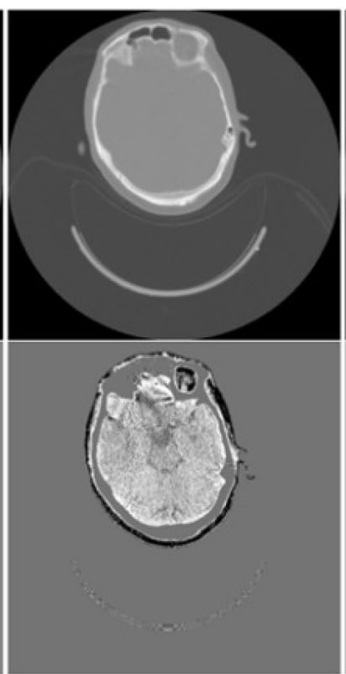

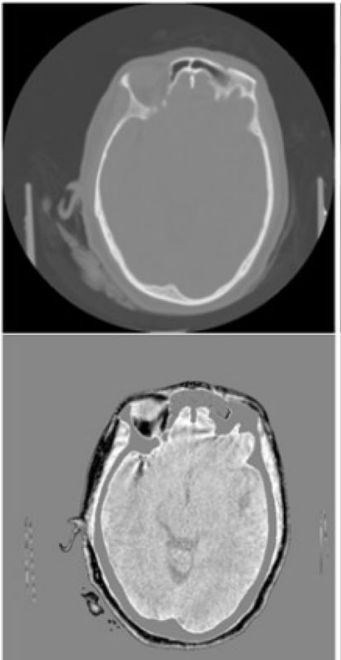

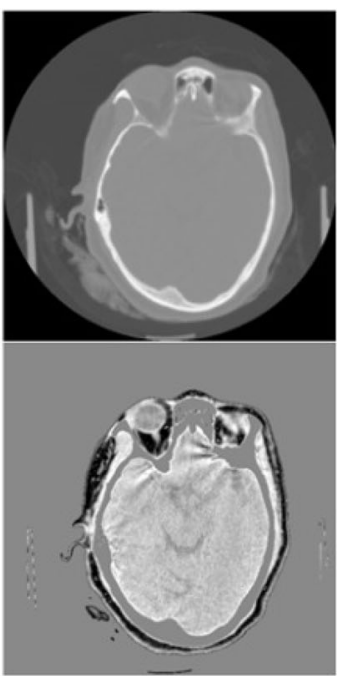

Fig. 3 Further example of enhancing via the proposed histogram equalization approach, a Input image, b Output images 
Fig. 4 Example of enhanced input images (a) and the LDN form of the improved image (b)
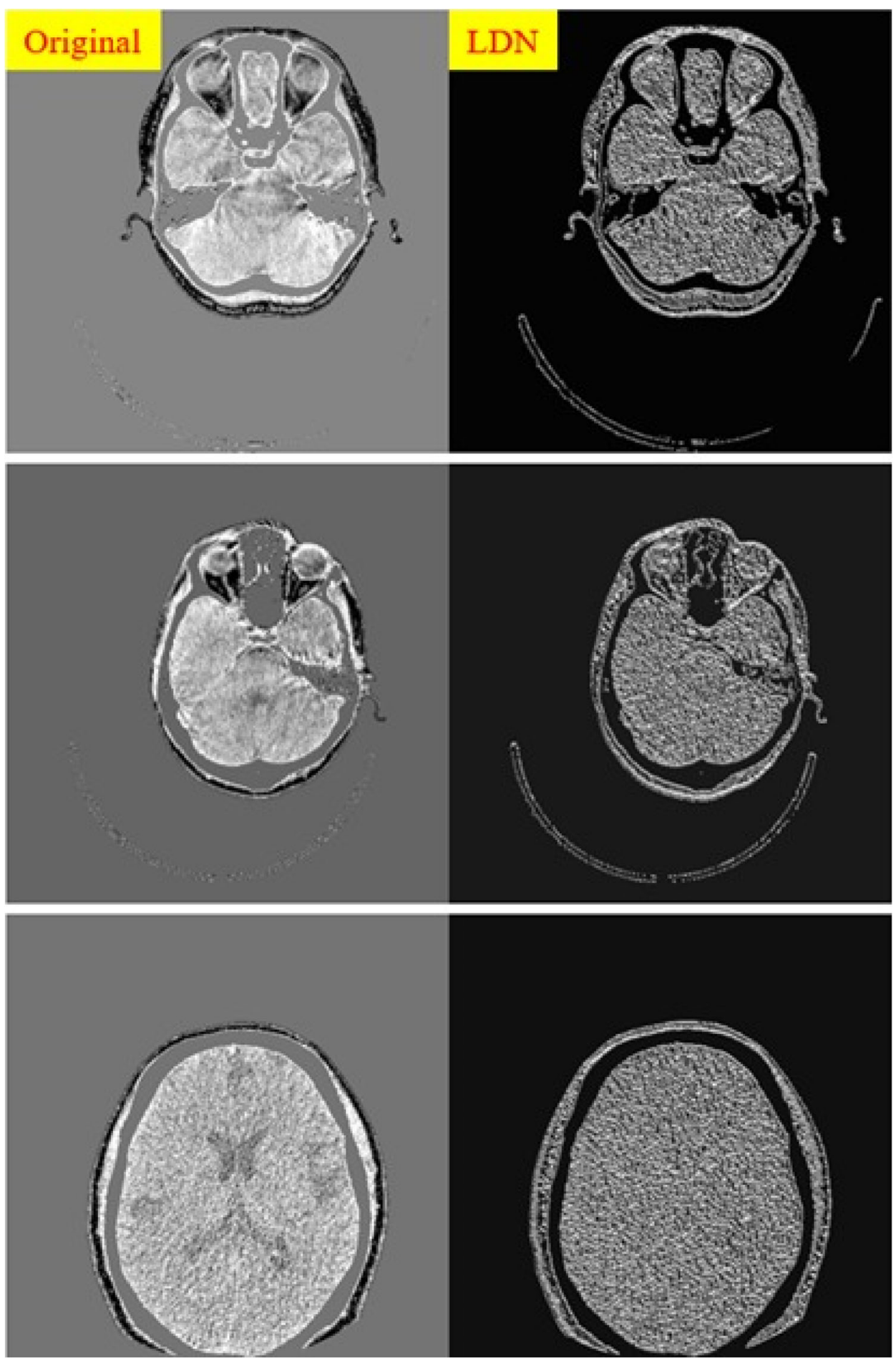

time to compute the dot product procedure. The convolutional layer can detect changes over a larger area with a broad receptive field, but the perception is less precise. The small receptive fields which lead to an accurate distinction between distinct regions are utilized in this study for detecting key features. The convolutional layer can detect stimuli over a specific region with a small receptive field, resulting in more accurate detection of small changes in the tumor boundary $[59,60]$.

To reduce the spatial dimension in the CNN architecture, the pooling layer is used. The pooling layers can decrease the spatial information, increase the computation performance, and reduce the possibility of the overfitting problem as the number of parameters decreases. Moreover, the pooling layer 


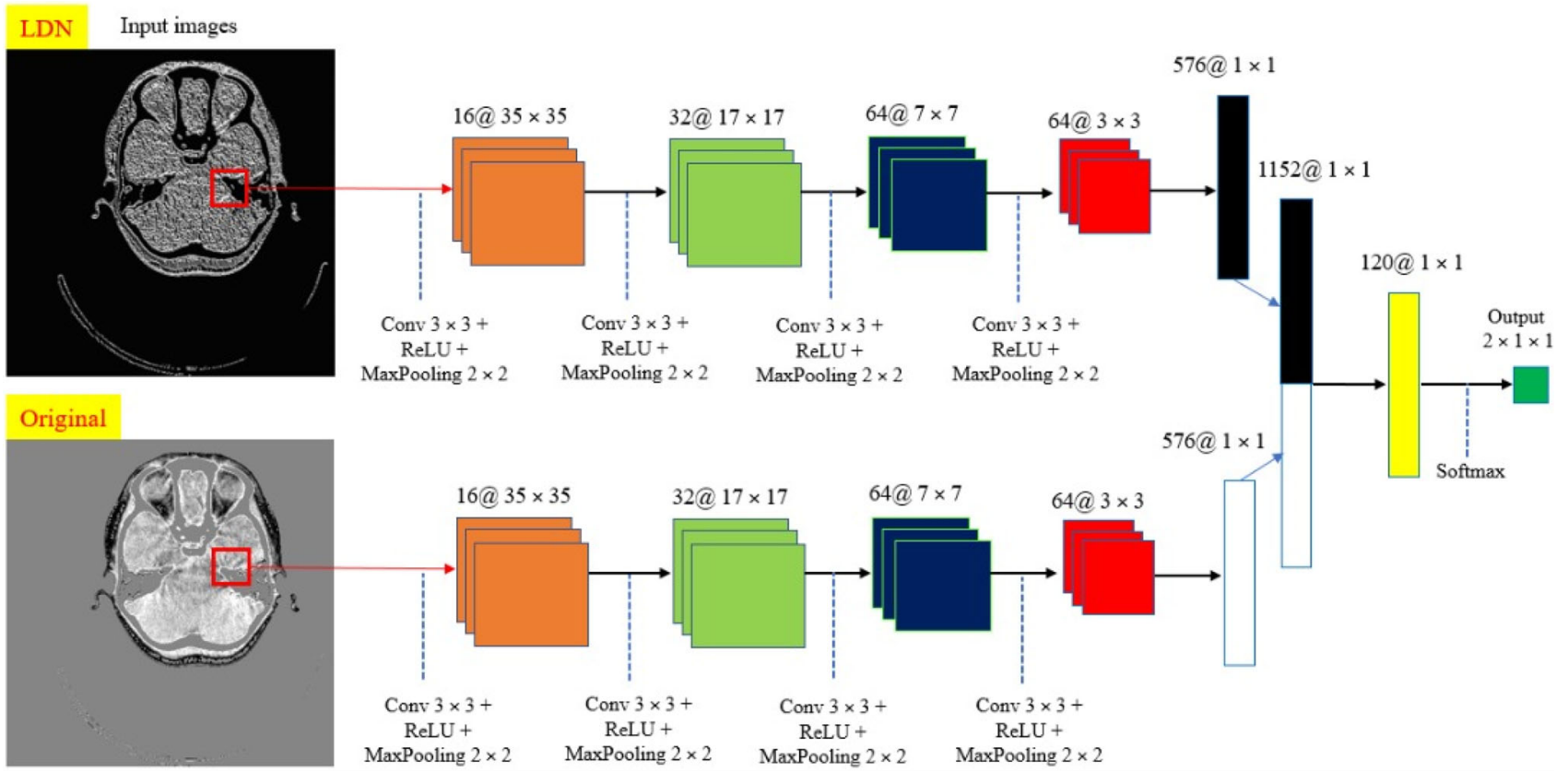

Fig. 5 The proposed $\mathrm{CNN}$ architecture

summarizes the features present in an area of the feature maps generated by some kernels in a convolution layer [61, 62]. On the other hand, selecting only suitable features from a tremendous number of generated features for object identification is done by the CNN architecture via activation layers. Activation layers are a critical part of designing a $\mathrm{CNN}$ model and the choice of activation function in the hidden layer will control how well the structure learns the training samples. The choice of activation function has a large impact on the capability and performance of the CNN model, and dissimilar activation functions may be employed in various parts of the structure [63, 64]. In this paper, we used MaxPooling and ReLU activation layers. This activation layer combats the vanishing gradient problem occurring in the sigmoid function [65]. Equation (10) demonstrates the ReLU function.

$f(x)=\max (0, x)$.

Figure 5 shows the proposed CNN structure. By using the proposed shallow $\mathrm{CNN}$ model, the dot product of the input images and the obtained binary mask are applied in both the input LDN image and the original image. Then, vital information is extracted for the effective distinction of the nerve optic and other tissues. As it is clearly shown in Fig. 5, there are two routes for extracting features of the LDN image and improved original image. At the end of routes, all features are merged to create a 1D vector. In this study, for all pixels inside the images, a patch with the size of $35 \times 35$ is selected for each input image. The center of the patch is located in each pixel for classifying images into never optic and other tissue classes. This process is repeated for all pixels inside the images. Convolutional layers capture spatial and temporal dependencies, and the output of a convolution is known as a feature map. The convolutional operation is the multiplying operation that computes the dot product between the filter with any receptive field and related pixels in the picture [66-68]. After each convolutional layer, a MaxPooling layer has been applied. This spatial pooling layer (downsampling layer) by selecting the maximum illumination value inside a $2 \times 2$ window is responsible for dimension reduction of the previous feature matrix obtained in the former layer (convolutional layer).

After extracting features and creating feature maps from each image, these 2D feature maps from each route are changed to a 1D feature map and put together to form a new 1D feature vector. A key layer for obtaining more highlevel features is the Fully-Connected layer (FC) that is placed before the classification output of a CNN. The last layer of the cascade $\mathrm{CNN}$ architecture that produces outputs with a probabilistic meaning is the Softmax regression layer. This layer is a form of logistic regression. The parameters employed for training the suggested pipeline are demonstrated in Table 1. The time of the features learning increases if small values are applied. Various values for the learning rate were applied to achieve the best classification performance while training the structures. As our model woks on the obtained patches rather than the whole image, it takes longer time for both training $(72 \mathrm{~h}$ ) and testing (around $5 \mathrm{~s}$ for each slice) process in compared to the applying whole image to A CNN model. 
Table 1 Parameters utilized for training the proposed pipeline

\begin{tabular}{ll}
\hline Parameters & Value \\
\hline Patch size & $35 \times 35$ \\
Activation function & Softmax \\
Output classes & 2 \\
Batch size & 10,000 \\
Learning rate drop factor & 0.35 \\
Max epochs & 120 \\
Learning rate & 0.001 \\
Optimizer & Adam \\
\hline
\end{tabular}

\section{Experimental results and discussion}

In this study, Dice, Precision, and Specificity are the scores used to assess the accuracy and efficiency of the proposed model. The Dice score is a validated reproducibility metric and a spatial overlap index that measures how well our model matched the hand-annotated ground truth training dataset segmentation to the expected labels $[69,70]$ and its formula is as follows:

Dice $=\left(2 \times\left(\frac{\mathrm{TP}}{2 \mathrm{TP}+\mathrm{FP}+\mathrm{FN}}\right)\right) \times 100 \%$.

The next index, Specificity means the proportion of correctly predicted other tissues concerning the total number of predicted other tissues.

Specificity $=\left(\frac{\mathrm{TN}}{\mathrm{TN}+\mathrm{FP}}\right)$.

The Precision index illustrates the proportion of correctly predicted nerve optic to total predicted nerve optic.

Precision $=\left(\frac{\mathrm{TP}}{\mathrm{TP}+\mathrm{FP}}\right)$,

where the FN, TN, FP, and TP imply False Negative, True Negative, False Positive, and True Positive, respectively.

In this paper, a public dataset was used from The Cancer Imaging Archive (TCIA) [28, 71]. TCIA is a service that hosts a large archive of medical images of cancer accessible for public download. We used $1600 \mathrm{CT}$ screening images and each volume dimension of these images is $512 \times 512$ $\times 35$. The annotation and interpretation of the dataset were done by two neuro-radiologists and the needed information was extracted from the CT screening images for ICP recognition. The experimental results were obtained by employing MATLAB 2019b over CUDA 9.0 and CuDNN 5.1 on Intel Core I7-3.2 GHz, 16 GB RAM, 8 MB Cache, and GPU 1050Ti NVIDIA computer under a 64-bit operating system.
Table 2 Comparative outcomes of the proposed strategy and recently published studies

\begin{tabular}{llll}
\hline Article name & $\begin{array}{l}\text { Dice } \\
(\%)\end{array}$ & Specificity (\%) & Precision (\%) \\
\hline FWHM [73] & 39.8 & 65.3 & 70.1 \\
$\begin{array}{l}\text { AUTONoMA } \\
\text { [75] }\end{array}$ & 52.4 & 75.3 & 71.5 \\
$\begin{array}{l}\text { Clustering [74] } \\
\begin{array}{l}\text { Deep learning } \\
\text { [72] }\end{array}\end{array}$ & 56.6 & 73.3 & 84.8 \\
$\begin{array}{l}\text { label-free 3D } \\
\text { segmentation }\end{array}$ & 75.9 & 79.4 & 82.1 \\
$\quad$ [76] & & & 85.7 \\
$\begin{array}{l}\text { Proposed } \\
\text { approach }\end{array}$ & 87.7 & 91.3 & \\
\hline
\end{tabular}

In this section, the performance of the proposed approach is compared with the other similar studies. The techniques employed in other studies for ICP include Deep learning [72], FWHM [73], Clustering [74], AUTONoMA [75], and labelfree 3D segmentation [76].

In this study, the efficiency of different methods is evaluated based on the three main values. We randomly divided our data samples into training data $(80 \%)$, test data $(10 \%)$, and validation data (10\%). Table 2 shows the quantitative value of Dice, Specificity, and Precision in each technique applied on the test samples. As highlighted, the proposed approach has the highest Specificity, Precision, and Dice values in comparison to other techniques which are reported $91.3 \%, 90.1 \%$, and $87.7 \%$, respectively. Next, the label-free 3D segmentation [76] has the highest value of Dice and Precision and Deep learning [72] has the highest value of specificity among five other approaches.

The FWHM [73] technique has the lowest Dice and Specificity value which makes it an inefficient technique to use for segmentation. The second method, AUTONoMA [75], has higher Dice and Specificity in comparison with FWHM; however, the value of Precision still is unacceptable. Based on the Clustering method, it is obvious that values of Dice and Precision have a considerable difference from the two previous methods. However, the Specificity reported $72.8 \%$ in which is more than FWHM and less than AUTONoMA approaches. In the Deep learning method, the value of Dice and Specificity increased considerably. However, the Precision value is two points lower than the clustering. Overall, the structures focused on Clustering and Deep learning methods have performed well but the networks' drawback is the need for a large dataset. Given the greater variability in the input images, these models may or may not work accurately. The next approach, label-free 3D is an efficient approach among the five previous segmentation techniques [76]. Even 
though the Dice and the Precision values have a considerable difference in comparison with the other methods, still is not efficient enough because of low Specificity. In the proposed approach, all criteria are improved and the value of the three-parameter shows the efficiency of this method which can substitute the other methods because of high yields and reliable results in segmentation. In the proposed approach, due to the fact of ICP early detection and accurate classification of pixels in nerve optic objects with high precision, the proposed technique is the most suitable approach for this purpose.

In the following, Figs. 6, 7, and 8 show the boxplot of Dice, Specificity, and Precision in different methods. In Fig. 6, the comparison of the dice value is presented in six different approaches. In the proposed method, the variance is about 0.87 and the median (red line) is close to the maximum value of the box plot ( 0.9 value) which is the indication of the robustness of the algorithm and the highest dice value. The next method with a higher dice value is a label-free method, the median is close to 0.75 and the variance value is close to 0.1 . Therefore, the Label-free robustness and dice value are lower than the proposed approach but higher in comparison with the other four algorithms. On contrary, the FWHM has a lower variance and the median is close to 0.38 . So, this algorithm possesses the lowest reliability and robustness among the six methods. In the deep learning and AUTONoMA methods, the median values are less than our obtained value but still are higher than other methods. The difference in the median value of AUTONoMA and cluster has approximately the same value and is about 0.05 .

Based on Fig. 7, the specificity value in six different methods is compared. In the proposed approach the median value is close to 0.92 which is an indication of the high reliability of this algorithm. Also, the variance value in this approach is about 0.05 . Therefore, the proposed approach has the highest value of specificity among the five other methods. In the deep learning method, the median value is close to the label-free approach and is about 0.81 and 0.79 , respectively. However, the variance value in the label-free technique is lower than the deep learning. So, the robustness of the algorithm is higher in the label-free approach. In two other methods, AUTONoMA and clustering, the median is about 0.75 and has an adjacent value of specificity. Among six different approaches, the FWHM possesses the lowest value of specificity. The median value is close to 0.04 and the variance is more than 0.01 .

Figure 8, shows the box plot of precision in different methods. In the proposed approach, the value of the median is close to 0.91 and possesses the highest value of precision and robustness. Next, label-free, clustering, and deep learning have a similar median value which is close to 0.85 . Therefore, these methods have similar robustness based on the precision index. However, the variance value in the clustering method is less than label-free and deep learning approaches. On the other hand, the FWHM and AUTONoMA have lower values and the median is about 0.7 . So, based on the precision index, it can be concluded that the reliability of these methods is low and cannot yield a robust result.

In the following, Fig. 9 demonstrates the CT screening images of three different patients. The results of detecting nerve optics using various techniques and the accuracy of detection are presented in this figure. As mentioned before, the recognition accuracy is highly depending on the quality of the image. So, the final results influence by both image enhancement and feature extraction techniques. In Fig. 9, each slice of CT images in three different columns is belonging to three different patients and the results of nerve optic detection with various methods are shown. The optic nerve starts at the optic disk, a structure that is $0.06 \mathrm{inch}(1.5 \mathrm{~mm})$ in diameter and is localized at the back of the eyeball. The goal in these slices of CT images is to detect the nerve optic behind the eyeball the different methods. Moreover, it is essential to define the angle of these detected nerves behind the eyeballs. It should be mentioned that, in much of the slices, only one nerve is demonstrated and can be recognized. As shown in Fig. 9, in column one, the left nerve optic is supposed to be detected correctly and right nerve is not represented clearly to be recognized. In the first column, as the left nerve is represented clearly, all approaches are able to detect the nerve optic appropriately. But, this is not true when we are dealing with an unclear border of the nerve optic (see third column).

In the proposed approach, the high dice, precision, and specificity were obtained and the never optics were recognized in the right direction. However, in the other methods, the straight line is detected on nerve optic, but the correct angle and distance are not detected. For instance, in the 'FWHM' method, the angle is not detected and the red line is near to the retina. The same problem does exist in the other models include 'AUTONoMA', 'Clustering', 'Deep learning', and 'Label-Free'.

In the second column, the left nerve optic is not detectable since it is not represented in this slice. However, in the right eye, the models try to detect the vertical lines beneath the retina. Therefore, other vertical nerves and muscles are detected in this area via 'FWHM', 'AUTONoMA', 'Clustering', 'Deep learning', and 'Label-Free' methods wrongly. However, in the proposed approach, the nerve optic is detected with high specificity and precision. Finally, in the third column, the methods that could identify left and right nerve optic correctly is the proposed method, 'Clustering', 'Deep learning', and 'Label-Free' approach. Nevertheless, the distance from the retina and the angle are not precise in the other methods. Overall, it can be concluded that the proposed approach is the best methodology to detect the nerve optic in comparison to the other method. Then, label-free, 
Fig. 6 Box plot showing the Dice in different methods

Fig. 7 Box plot showing the Specificity in different methods
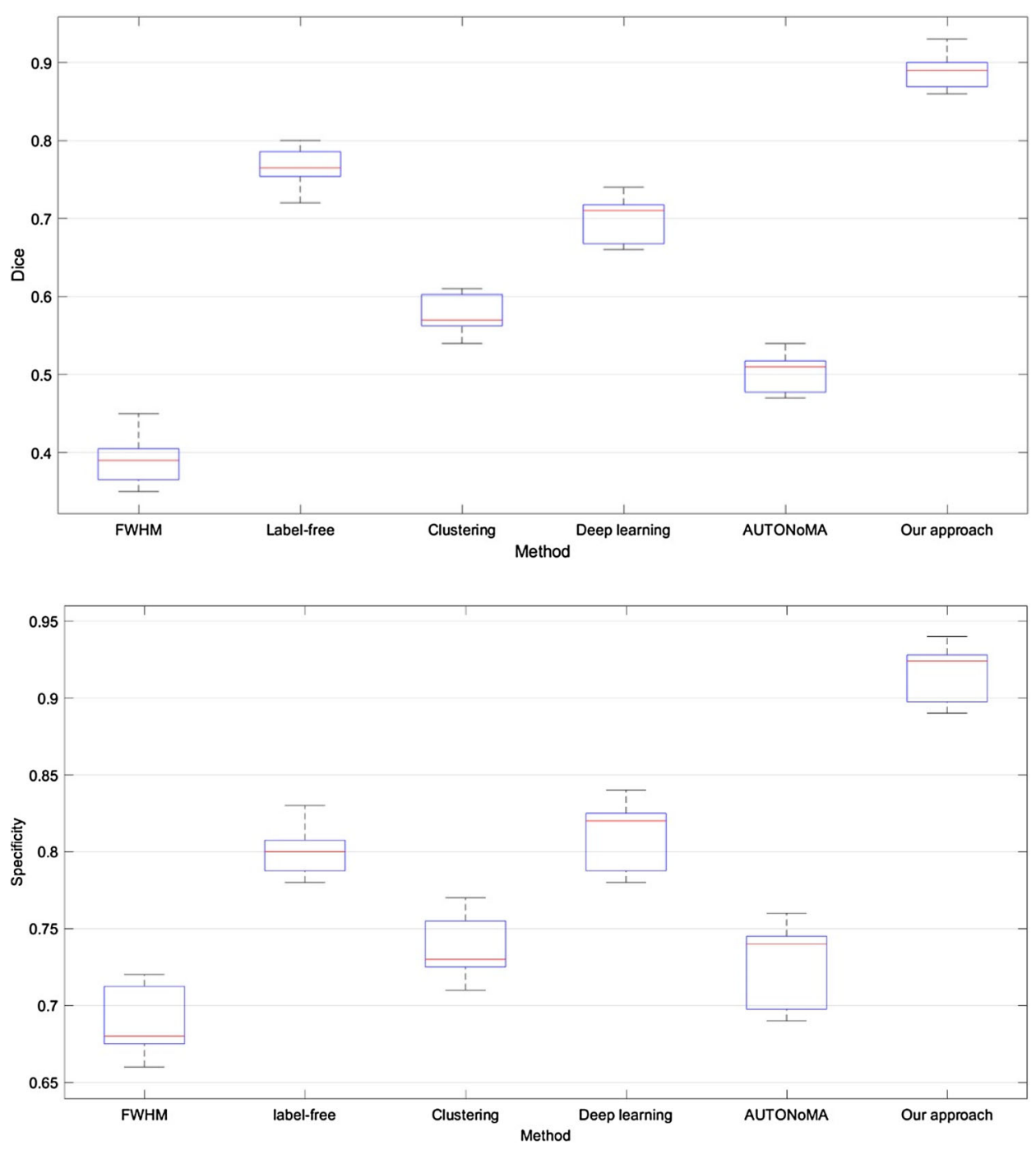

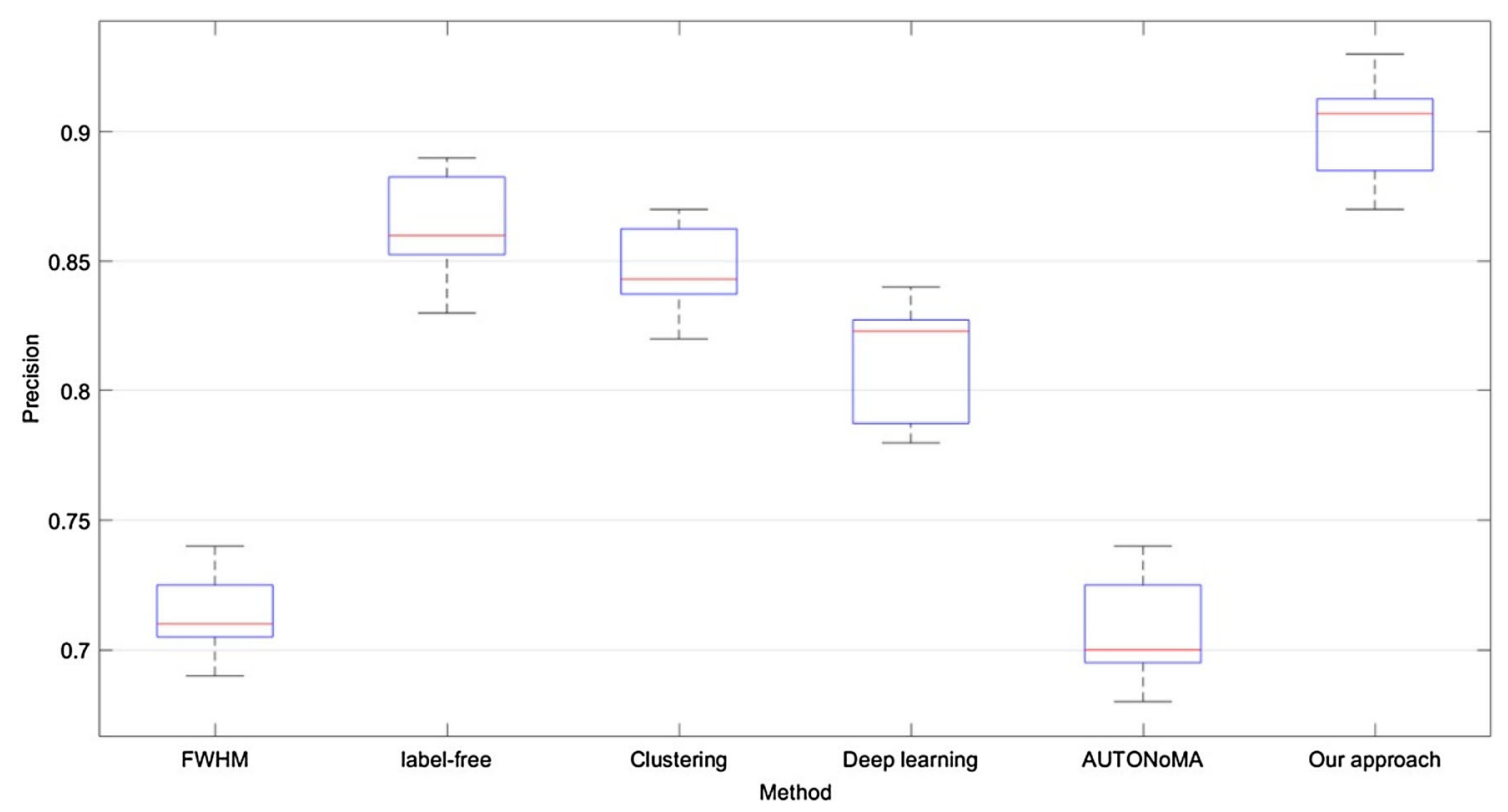

Fig. 8 Box plot showing the precision in different methods 


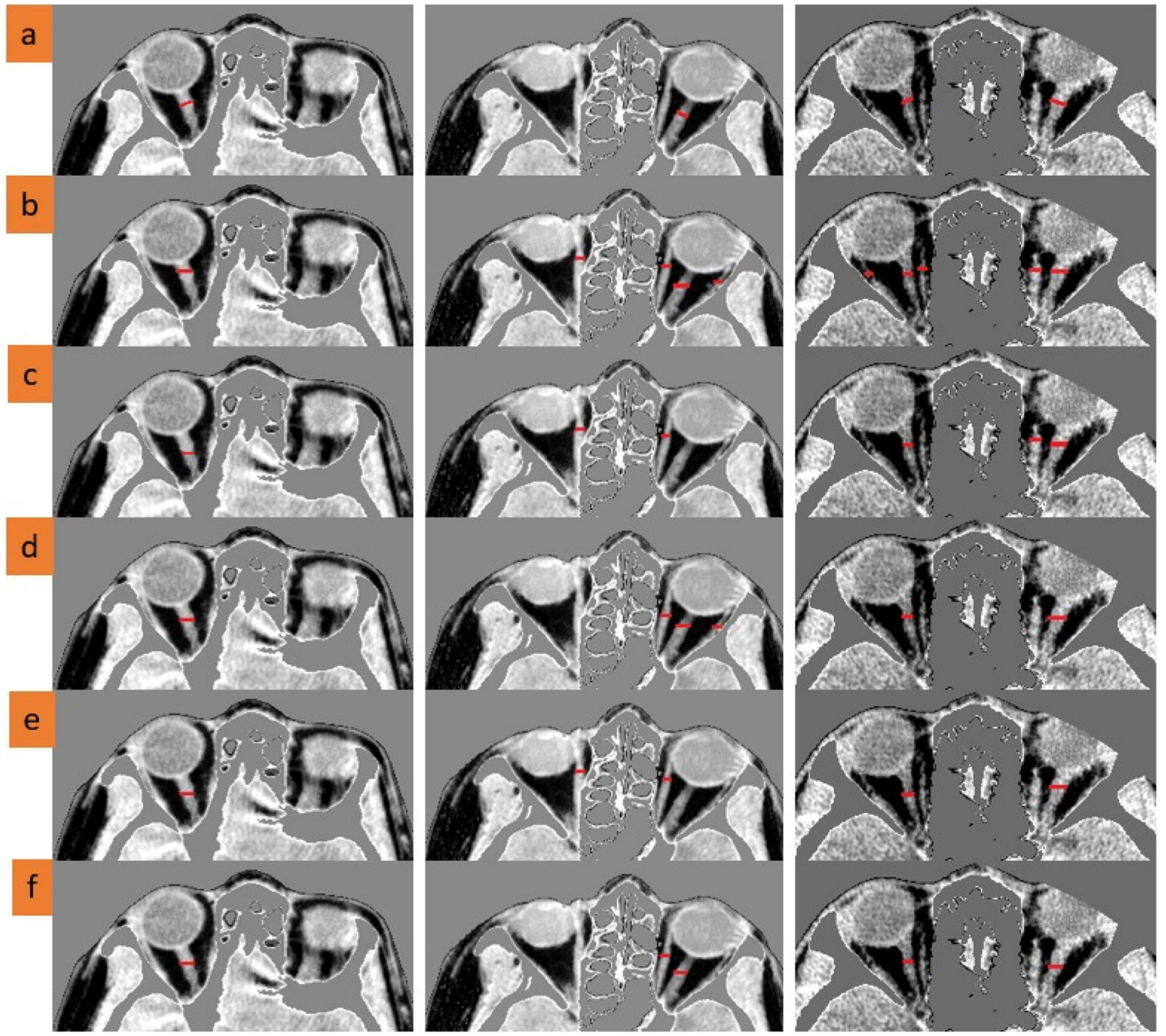

Fig. 9 The results of nerve optic segmentation using a The proposed approach, b FWHM, c AUTONoMA, d Clustering, e Deep learning, $\mathbf{f}$ Label free

deep learning, and clustering methods, respectively, provided better detection of nerve optic.

As it is clearly demonstrated, the Deep learning method [72] seems to perform better than the Clustering method [74] in detecting vertical objects (like Nerve Optic), whereas the proposed method is much better not only in detecting such areas but also can eliminate unrelated vertical objects more accurately. Also, the proposed approach, Label-Free 3D segmentation, and the deep learning methods gain promising results in detecting the optic nerve between two dark areas.

\section{Conclusion}

This study presents an approach based on the CNN technique for the nerve optic accurate segmentation in the brain CT screening images. The slope of the nerve optic line and its distance behind the eyeball is the main issue for correct detection of the nerve optic. A new region-based strategy was used for improving the quality of the input image. This technique is applied only in some important parts of the image and insignificant parts of the image declined by the CNN model. 
By utilizing a textural descriptor method, LDN, a new image was produces based on the improved images and represents more detail about the eyeball and nerve optic. Next, in any stage of CNN, two obtained images are utilized for feature extraction and classification steps. Due to using the LDN approach and extracting some other vital information, the training process of the network does not require a large amount of data.

Based on the results, the output classification of the proposed $\mathrm{CNN}$ is close to human observation and the comparison of the different techniques such as Clustering, FWHM, AUTONoMA, deep learning, and label-free 3D segmentation shows that the proposed approach outperforms and yields results with high value of dice, specificity, and precision in which the values reported $87.7 \%, 91.3 \%$, and $90.1 \%$, respectively. As compared to recently published frameworks, an extensive investigation has demonstrated the efficacy of the proposed method.

The suggestion for future studies is to use a powerful pre-processing method that can be able to remove the majority of insignificant parts of an image before applying it to a CNN model. This is due to the fact that without representing the nerve optic in an appropriate way, extracting informative features is not possible. Also, to overcome the problem of insufficient training samples, some augmentation approached can be applied to increase the training data.

Funding This research received no specific grant from any funding agency in the public, commercial, or not-for-profit sectors.

Availability of data and materials In this paper, a public dataset was used from The Cancer Imaging Archive (TCIA) [28, 71]. TCIA is a service which hosts a large archive of medical images of cancer accessible for public download.

\section{Declarations}

Conflict of interest The authors declare that they have no known competing financial interests or personal relationships that could have appeared to influence the work reported in this paper.

Ethics approval The manuscript in part or in full has not been submitted or published anywhere. In other words, the authors should ensure that the manuscript is not a duplicate publication.

Open Access This article is licensed under a Creative Commons Attribution 4.0 International License, which permits use, sharing, adaptation, distribution and reproduction in any medium or format, as long as you give appropriate credit to the original author(s) and the source, provide a link to the Creative Commons licence, and indicate if changes were made. The images or other third party material in this article are included in the article's Creative Commons licence, unless indicated otherwise in a credit line to the material. If material is not included in the article's Creative Commons licence and your intended use is not permitted by statutory regulation or exceeds the permitted use, you will need to obtain permission directly from the copyright holder. To view a copy of this licence, visit http://creativecomm ons.org/licenses/by/4.0/.

\section{References}

1. Canac N, Jalaleddini K, Thorpe SG, Thibeault CM, Hamilton RB (2020) Review: pathophysiology of intracranial hypertension and noninvasive intracranial pressure monitoring. Fluids Barriers CNS 17(1):40. https://doi.org/10.1186/s12987-020-00201-8

2. Soroushmehr R et al. (2019) Automated optic nerve sheath diameter measurement using super-pixel analysis. In: Proceedings of the annual international conference of the IEEE engineering in medicine and biology society, EMBS, pp 2793-2796. https://doi. org/10.1109/EMBC.2019.8856449

3. Xu W, Gerety P, Aleman T, Swanson J, Taylor J (2016) Noninvasive methods of detecting increased intracranial pressure. Child's Nerv Syst 32(8):1371-1386. https://doi.org/10.1007/s00381-0163143-x

4. Pitfield AF, Carroll AB, Kissoon N (2012) Emergency management of increased intracranial pressure. Pediatr Emerg Care 28(2):200-204. https://doi.org/10.1097/PEC.0b013e318243fb72

5. Lee SB et al (2020) Artifact removal from neurophysiological signals: impact on intracranial and arterial pressure monitoring in traumatic brain injury. J Neurosurg 132(6):1952-1960. https://doi. org/10.3171/2019.2.JNS182260

6. Little RD (2008) Increased intracranial pressure. Clin Pediatr Emerg Med 9(2):83-87. https://doi.org/10.1016/j.cpem.2008. 03.003

7. Raboel PH, Bartek J, Andresen M, Bellander BM, Romner B (2012) Intracranial pressure monitoring: invasive versus non-invasive methods-a review. Crit Care Res Pract 2012:14. https://doi.org/10. 1155/2012/950393

8. Robba C et al (2018) Optic nerve sheath diameter measured sonographically as non-invasive estimator of intracranial pressure: a systematic review and meta-analysis. Intensive Care Med 44(8):1284-1294. https://doi.org/10.1007/s00134-018-5305-7

9. Wang LJ et al (2018) Ultrasonography assessments of optic nerve sheath diameter as a noninvasive and dynamic method of detecting changes in intracranial pressure. JAMA Ophthalmol 136(3):250-256. https://doi.org/10.1001/jamaophthalmol. 2017.6560

10. Kim DH, Jun JS, Kim R (2018) Measurement of the optic nerve sheath diameter with magnetic resonance imaging and its association with eyeball diameter in healthy adults. J Clin Neurol 14(3):345-350. https://doi.org/10.3988/jen.2018.14.3.345

11. Bäuerle J, Nedelmann M (2012) B-mode sonography of the optic nerve in neurological disorders with altered intracranial pressure. Perspect Med 1-12(1-12):404-407. https://doi.org/10.1016/ j.permed.2012.01.001

12. Wang LJ, Chen HX, Chen Y, Yu ZY, Xing YQ (2020) Optic nerve sheath diameter ultrasonography for elevated intracranial pressure detection. Ann Clin Transl Neurol 7(5):865-868. https://doi.org/ 10.1002/acn3.51054

13. Harrigan RL et al (2017) Quantitative characterization of optic nerve atrophy in patients with multiple sclerosis. Mult Scler J Exp Transl Clin. https://doi.org/10.1177/2055217317730097

14. Pang $\mathrm{M}$ et al (2019) Measurement of Optic nerve sheath on ocular ultrasound image based on segmentation by CNN. https://doi.org/ 10.1109/ICSIDP47821.2019.9173198.

15. Naldi A et al (2020) Optic nerve sheath diameter asymmetry in healthy subjects and patients with intracranial hypertension. Neurol Sci 41(2):329-333. https://doi.org/10.1007/s10072-019-04076-y

16. Zhu S, Cheng C, Zhao D, Zhao Y, Liu X, Zhang J (2021) Clinical value of optic nerve sheath diameter assessment in prognosis of comatose patients with supratentorial lesions. https://doi.org/10. 21203/RS.3.RS-186293/V1 
17. Comaniciu D, Meer P (2002) Cell image segmentation for diagnostic pathology. Advanced algorithmic approaches to medical image segmentation. Springer, London, pp 541-558

18. Dorosti S, Jafarzadeh Ghoushchi S, Sobhrakhshankhah E, Ahmadi M, Sharifi A (2020) Application of gene expression programming and sensitivity analyses in analyzing effective parameters in gastric cancer tumor size and location. Soft Comput 24(13):9943-9964. https://doi.org/10.1007/s00500-019-04507-0

19. Ranjbarzadeh R, Saadi SB (2020) Automated liver and tumor segmentation based on concave and convex points using fuzzy c-means and mean shift clustering. Meas J Int Meas Confed 150:107086. https://doi.org/10.1016/j.measurement.2019.107086

20. Brata Chanda P, Sarkar SK (2019) Automatic identification of blood vessels, exaudates and abnormalities in retinal images for diabetic retinopathy analysis. SSRN Electron J. https://doi.org/10. 2139/ssrn.3446607

21. Senders JT et al (2018) An introduction and overview of machine learning in neurosurgical care. Acta Neurochir 160(1):29-38. https://doi.org/10.1007/s00701-017-3385-8

22. Ojeda P, Zawaideh M, Mossa-Basha M, Haynor D (2019) The utility of deep learning: evaluation of a convolutional neural network for detection of intracranial bleeds on non-contrast head computed tomography studies. 10949:128. https://doi.org/10.1117/12. 2513167

23. Raj R et al (2019) Machine learning-based dynamic mortality prediction after traumatic brain injury. Sci Rep 9(1):1-13. https://doi. org/10.1038/s41598-019-53889-6

24. Naraei P, Nouri M, Sadeghian A (2018) Toward learning intracranial hypertension through physiological features: a statistical and machine learning approach. In: 2017 intelligent systems conference, IntelliSys 2017, vol 2018-Janua, pp 395-399. https://doi.org/ 10.1109/IntelliSys.2017.8324324

25. Chen W, Cockrell C, Ward KR, Najarian K (2010) Intracranial pressure level prediction in traumatic brain injury by extracting features from multiple sources and using machine learning methods. In: Proceedings - 2010 IEEE international conference on bioinformatics and biomedicine, BIBM 2010, pp 510-515. https://doi.org/ 10.1109/BIBM.2010.5706619

26. Hu X, Xu P, Asgari S, Vespa P, Bergsneider M (2010) Forecasting ICP elevation based on prescient changes of intracranial pressure waveform morphology. IEEE Trans Biomed Eng 57(5):1070-1078. https://doi.org/10.1109/TBME.2009.2037607

27. Quachtran B, Hamilton R, Scalzo F (2016) Detection of intracranial hypertension using deep learning. Proc Int Conf Pattern Recogn. https://doi.org/10.1109/ICPR.2016.7900010

28. Search. https://public.cancerimagingarchive.net/nbia-search/. Accessed 13 Jun 2021

29. Dunn JC (1973) A fuzzy relative of the ISODATA process and its use in detecting compact well-separated clusters. J Cybern 3(3):32-57. https://doi.org/10.1080/01969727308546046

30. Bezdek JC (1981) Objective function clustering. Pattern recognition with fuzzy objective function algorithms. Springer, US, pp 43-93

31. Ranjbarzadeh R, Saadi SB, Amirabadi A (2020) LNPSS: SAR image despeckling based on local and non-local features using patch shape selection and edges linking. Meas J Int Meas Confed. https://doi.org/10.1016/j.measurement.2020.107989

32. Pourasad Y, Ranjbarzadeh R, Mardani A (2021) A new algorithm for digital image encryption based on chaos theory. Entropy 23(3):341. https://doi.org/10.3390/e23030341

33. Bendechache M, Kechadi MT, Le-Khac NA (2016) Efficient large scale clustering based on data partitioning. In: Proceedings-3rd IEEE international conference on data science and advanced analytics, DSAA 2016, pp 612-621. https://doi.org/10.1109/DSAA. 2016.70
34. Hamzenejad A, Ghoushchi SJ, Baradaran V, Mardani A (2020) A robust algorithm for classification and diagnosis of brain disease using local linear approximation and generalized Autoregressive Conditional Heteroscedasticity model. Mathematics 8(8):1268. https://doi.org/10.3390/MATH8081268

35. Bendechache M (2019) Study of distributed dynamic clustering framework for spatial data mining. http://oatd.org/oatd/record? record $=$ handle $\% 5$ C\%3A10197\%5C\%2F10614. Accessed 17 Dec 2020

36. Bendechache M, Kechadi MT (2015) Distributed clustering algorithm for spatial data mining. In: ICSDM 2015-proceedings 2015 2nd IEEE international conference on spatial data mining and geographical knowledge services, pp 60-65. https://doi.org/10.1109/ ICSDM.2015.7298026

37. Deng X (2018) Enhancing image quality via style transfer for single image super-resolution. IEEE Signal Process Lett 25(4):571-575. https://doi.org/10.1109/LSP.2018.2805809

38. Kaur P, Singh H, Gupta A, Girdhar A (2014) An improved steganographic approach to diminish data modification for enhancing image quality. In: 2014 International conference on medical imaging, m-health and emerging communication systems, MedCom 2014, pp 329-333. https://doi.org/10.1109/MedCom.2014. 7006027

39. Zhou Y, Zhang T, Zhong F, Guo S (2019) Enhancing image quality of ghost imaging by fuzzy c-means clustering method. AIP Adv 9(7):75006. https://doi.org/10.1063/1.5079681

40. Sunitha TO, Rajalakshmi R (2020) Multi-modal image fusion technique for enhancing image quality with multi-scale decomposition algorithm. Comput Methods Biomech Biomed Eng Imaging Vis. https://doi.org/10.1080/21681163.2020.1830437

41. Ge $\mathrm{Y}$ et al (2020) Enhancing the X-ray differential phase contrast image quality with deep learning technique. IEEE Trans Biomed Eng. https://doi.org/10.1109/tbme.2020.3011119

42. Singh K, Kapoor R (2014) Image enhancement using exposure based sub image histogram equalization. Pattern Recognit Lett 36(1):10-14. https://doi.org/10.1016/j.patrec.2013.08.024

43. Celik T (2012) Two-dimensional histogram equalization and contrast enhancement. Pattern Recognit 45(10):3810-3824. https:// doi.org/10.1016/j.patcog.2012.03.019

44. Zuo C, Chen Q, Sui X (2013) Range limited bi-histogram equalization for image contrast enhancement. Optik (Stuttg) 124(5):425-431. https://doi.org/10.1016/j.ijleo.2011.12.057

45. Patel S, Goswami M (2014) Comparative analysis of Histogram Equalization techniques. In: Proceedings of 2014 international conference on contemporary computing and informatics, IC3I 2014, pp 167-168. https://doi.org/10.1109/IC3I.2014.7019808

46. Michael Revina I, Sam Emmanuel WR (2018) Face expression recognition using LDN and dominant gradient local ternary pattern descriptors. J King Saud Univ Comput Inf Sci. https://doi.org/10. 1016/j.jksuci.2018.03.015

47. Ramírez Rivera A, Rojas Castillo J, Chae O (2015) Local directional texture pattern image descriptor. Pattern Recognit Lett 51:94-100. https://doi.org/10.1016/j.patrec.2014.08.012

48. Rivera AR, Castillo JR, Chae O (2013) Local directional number pattern for face analysis: face and expression recognition. IEEE Trans Image Process 22(5):1740-1752. https://doi.org/10.1109/ TIP.2012.2235848

49. Lo SCB, Chan HP, Lin JS, Li H, Freedman MT, Mun SK (1995) Artificial convolution neural network for medical image pattern recognition. Neural Netw 8(7-8):1201-1214. https://doi.org/10. 1016/0893-6080(95)00061-5

50. Mishkin D, Sergievskiy N, Matas J (2017) Systematic evaluation of convolution neural network advances on the Imagenet. Comput Vis Image Underst 161:11-19. https://doi.org/10.1016/j.cviu.2017. 05.007 
51. Hubel DH, Wiesel TN (1968) Receptive fields and functional architecture of monkey striate cortex. J Physiol 195(1):215-243. https:// doi.org/10.1113/jphysiol.1968.sp008455

52. Razmjooy N, Razmjooy S, Vahedi Z, Estrela VV, de Oliveira GG (2021) Skin color segmentation based on artificial neural network improved by a modified grasshopper optimization algorithm. In: Lecture notes in electrical engineering, vol 696. Springer Science and Business Media Deutschland GmbH, New York, pp 169-185

53. Ranjbarzadeh R, BagherianKasgari A, Jafarzadeh Ghoushchi S, Anari S, Naseri M, Bendechache M (2021) Brain tumor segmentation based on deep learning and an attention mechanism using MRI multi-modalities brain images. Sci Rep 11(1):10930. https:// doi.org/10.1038/s41598-021-90428-8

54. Ain QT et al (2017) Sentiment analysis using deep learning techniques: a review. IJACSA Int J Adv Comput Sci Appl 8(6):424

55. Wahab N, Khan A, Lee YS (2017) Two-phase deep convolutional neural network for reducing class skewness in histopathological images based breast cancer detection. Comput Biol Med 85:86-97. https://doi.org/10.1016/j.compbiomed.2017.04.012

56. Al-Saffar AAM, Tao H, Talab MA (2017) Review of deep convolution neural network in image classification. In: Proceeding-2017 international conference on radar, antenna, microwave, electronics, and telecommunications, ICRAMET 2017, vol 2018-Janua, pp 26-31. https://doi.org/10.1109/ICRAMET.2017.8253139

57. Wang J, Zhuang J, Duan L, Cheng W (2016) A multi-scale convolution neural network for featureless fault diagnosis. In: International Symposium on Flexible Automation, ISFA 2016, pp 65-70. https:// doi.org/10.1109/ISFA.2016.7790137

58. Liu CY, Sun WB, Chao WH, Che WX (2013) Convolution neural network for relation extraction. In: Lecture notes in computer science (including subseries lecture notes in artificial intelligence and lecture notes in bioinformatics), vol 8347 LNAI, no. PART 2, pp 231-242. https://doi.org/10.1007/978-3-642-53917-6_21

59. Huang Z, Wang Z, Zhang J, Li Q, Shi Y (2021) Image enhancement with the preservation of brightness and structures by employing contrast limited dynamic quadri-histogram equalization. Optik (Stuttg). 226:165877. https://doi.org/10.1016/j.ijleo.2020.165877

60. Mahmood A et al (2017) Deep learning for coral classification. Handbook of neural computation. Elsevier Inc., Amsterdam, pp 383-401

61. Sun M, Song Z, Jiang X, Pan J, Pang Y (2017) Learning pooling for convolutional neural network. Neurocomputing 224:96-104. https://doi.org/10.1016/j.neucom.2016.10.049

62. Yu D, Wang H, Chen P, Wei Z (2014) Mixed pooling for convolutional neural networks. In: Lecture notes in computer science (including subseries lecture notes in artificial intelligence and lecture notes in bioinformatics), vol 8818, pp 364-375. https://doi. org/10.1007/978-3-319-11740-9_34

63. Wang HX, Zhou JQ, Gu CH, Lin H (2019) Design of activation function in CNN for image classification. Zhejiang Daxue Xuebao (Gongxue Ban) J Zhejiang Univ Eng Sci 53(7):1363-1373. https:// doi.org/10.3785/j.issn.1008-973X.2019.07.016

64. Wang S, Wang Y, Wang D, Yin Y, Wang Y, Jin Y (2020) An improved random forest-based rule extraction method for breast cancer diagnosis. Appl Soft Comput J 86:105941. https://doi.org/ 10.1016/j.asoc.2019.105941

65. Ranjbarzadeh $\mathrm{R}$ et al (2021) Lung infection segmentation for COVID-19 pneumonia based on a cascade convolutional network from CT images. Biomed Res Int 2021:1-16. https://doi.org/10. $1155 / 2021 / 5544742$

66. de Assis Neto SR et al. (2020) Detecting human activities based on a multimodal sensor data set using a bidirectional long short-term memory model: a case study. In: Studies in systems, decision and control, vol 273. Springer, New York, pp 31-51
67. Frid-Adar M, Diamant I, Klang E, Amitai M, Goldberger J, Greenspan H (2018) GAN-based synthetic medical image augmentation for increased CNN performance in liver lesion classification. Neurocomputing 321:321-331. https://doi.org/10.1016/j.neucom. 2018.09.013

68. Tian Q, Wu Y, Ren X, Razmjooy N (2021) A New optimized sequential method for lung tumor diagnosis based on deep learning and converged search and rescue algorithm. Biomed Signal Process Control 68:102761. https://doi.org/10.1016/j.bspc.2021.102761

69. Karimi N, RanjbarzadehKondrood R, Alizadeh T (2017) An intelligent system for quality measurement of Golden Bleached raisins using two comparative machine learning algorithms. Meas J Int Meas Confed 107:68-76. https://doi.org/10.1016/j.measurement. 2017.05.009

70. Munir K, Elahi H, Ayub A, Frezza F, Rizzi A (2019) Cancer diagnosis using deep learning: a bibliographic review. Cancers (Basel) 11(9):1235. https://doi.org/10.3390/cancers 11091235

71. Clark K et al (2013) The cancer imaging archive (TCIA): maintaining and operating a public information repository. J Digit Imaging 26(6):1045-1057. https://doi.org/10.1007/s10278-013-9622-7

72. Zhu $F$ et al (2020) Semantic segmentation using deep learning to extract total extraocular muscles and optic nerve from orbital computed tomography images. http://arxiv.org/abs/2007. 02091. Accessed 20 Jan 2021

73. Chow LS, Teymouri M (2014) Measurement of human optic nerve's diameter using magnetic resonance imaging (MRI) images. https://doi.org/10.1109/ICIAS.2014.6869542

74. Rajajee V et al (2020) Novel algorithm for automated optic nerve sheath diameter measurement using a clustering approach. Mil Med. https://doi.org/10.1093/milmed/usaa231

75. Meiburger KM et al (2020) Automatic optic nerve measurement: a new tool to standardize optic nerve assessment in ultrasound Bmode images. Ultrasound Med Biol 46(6):1533-1544. https://doi. org/10.1016/j.ultrasmedbio.2020.01.034

76. Thiéry AH et al (2020) Towards label-free 3D segmentation of optical coherence tomography images of the optic nerve head using deep learning. Biomed Opt Express 11(11):6356-6378. https://doi. org/10.1364/BOE.395934

Publisher's Note Springer Nature remains neutral with regard to jurisdictional claims in published maps and institutional affiliations. 